\title{
Machine learning und humane Intelligenz
}

Yves C. A. Robert

Prof. Dr. med.

Der Artikel von Goldhahn et al. [1] spricht ein Thema an, welches nicht nur die Wissenschaft, sondern in kürzester Zeit auch den medizinischen Alltag gehörig umkrempeln wird. Man denke nur schon, wie die Verknüpfung moderner Bildverarbeitungsmethoden mit der künstlichen Intelligenz die Diagnostik visuell orientierter Fächer (Radiologie, Dermatologie Ophthalmologie) revolutionieren kann. Die Befürchtung, ob und wann die künstliche Intelligenz, wohl besser mit dem Terminus «machine learning» umschrieben, die natürliche Intelligenz (NI) überflügeln oder gar ersetzen wird, ist somit verständlich. Die Antwort auf diese Frage ist nur möglich beim direkten Vergleich der zwei Phänomene. Dazu muss bei der NI nicht nur die psychologisch-intellektuelle Dimension, sondern auch ihre physiologische Dimension in Betracht gezogen werden. Hier soll diese physiologische Dimension noch einmal in Erinnerung gerufen werden.

\section{Wechselwirkung mit der Umwelt}

Innerhalb der Lebenswelt ist Intelligenz vielfältig vorhanden. Das hängt damit zusammen, dass Leben in Wechselwirkung mit der Umwelt steht. (In Anlehnung an die Definition von Karl Popper: «Leben ist ein dauerndes Problemelösen.») In den «niederen» Lebensformen

\section{Résumé}

Pour évaluer si l'intelligence artificielle constitue une menace ou un atout pour l'activité médicale, il faudrait pouvoir la comparer directement avec l'intelligence humaine. C'est ce que nous tentons de faire dans le présent article, en nous inspirant d'une conception proche de la physiologie sensorielle, avec un modèle de robot de type input-traitement-output. II s'avère que l'intelligence humaine est fortement marquée par l'input et l'output, à savoir par les organes sensoriels et par les organes qui agissent, alors que dans I'intelligence artificielle, le "traitement", à savoir le calcul, joue le rôle principal. Cet aspect, avec d'autres particularités, empêche une comparaison directe des deux formes d'intelligence. On peut donc en conclure que l'arrivée de l'intelligence artificielle dans le quotidien médical ne concurrence pas la pensée spécifiquement médicale. wirken physikalische und chemische Reize direkt auf den Organismus. Bestimmte Substanzen diffundieren durch die Zellwand des Einzellers und lösen in der Zelle einen chemischen Prozess aus, der eine Reaktion hervorruft. Je nach Situation kann der Organismus die Reaktion modifizieren. In den «höheren» Lebensformen ist die NI an die Entwicklung eines Nervensystems gebunden. Bei den Säugetieren wird chemische und physikalische Energie aus der Umwelt über die sechs natürlichen Sinne aufgenommen, in einen Reiz umgewandelt und via Nervensystem dem Gehirn weitergeleitet. Die Antwort erfolgt über die Nerven motorisch an die Muskeln. Diese Anordnung ist tatsächlich vergleichbar mit einem Roboter nach dem Schema: Input-Verarbeitung-Output. Im Verlaufe der Evolution sind so Reflexe, Instinkte, Gefühle entstanden. Auch bei der Intelligenz, die den Menschen in den letzten 150000 Jahren von den anderen Primaten entfernt hat (humane Intelligenz [HI]), lässt sich ihre Leistung am Output beurteilen. Im Folgenden soll das oben angesprochene Schema Input-VerarbeitungOutput unter besonderer Berücksichtigung dreier Problemkreise auf die humane Intelligenz angewendet werden. 


\section{Input - Sensor}

Der Input beim Menschen erfolgt über die Sinnesorgane, sie sind der Sensor. Nach Umwandlung der Umweltenergie im Sinnesorgan wird ein Signal (Nervenimpuls) an das Gehirn gesandt, was eine Empfindung und, je nach Fall, eine Wahrnehmung auslöst. Wahrnehmung bedeutet hier Verknüpfung mit dem Bewusstsein. Beim Gesichtssinn wird elektromagnetische Strahlung mit Wellenlängen von 400-800 nm von der Netzhaut absorbiert. Das Signal liefert dem Gehirn nicht Wellenlängen, sondern die 7 Informationen: Farbe, zeitliche Auflösung (frame rate), örtliche Auflösung (Trennschärfe), Bewegung, Adaptation (Anpassung an die Helligkeit der Umwelt) und Kontrast. Die siebte Information, das Zusammenfügen der seitlich versetzten Bilder der zwei Augen zu einem einzigen dreidimensionalen Bild (Fusion), ist eine beidäugige Informationsverarbeitung im Gehirn. Beim Gehör bewirkt das Signal aus Schallwellen mit Frequenzen von ca. 18-20 000 Hz die Information Ton, Klang, Geräusch, Lärm. Die Bestimmung der Schallrichtung ist eine binaurale Informationsverarbeitung im Gehirn. Beim Gleichgewicht wird das Signal als Beschleunigung wahrgenommen. Die haptischen Sinne oder Hautsinne übermitteln warm/kalt, Berührung, Druck, Schmerz, Kitzel, Juckreiz. Und die chemischen Sinne liefern als Geschmack süss, salzig, bitter, sauer (umami) plus über die Nase eine Vielzahl von Gerüchen. Diese sechs natürlichen Sinne formen unsere Welt, eine Welt, die nicht der physikalischen Welt entspricht. Sie ist zudem spezifisch für den Menschen. Für jedes andere Lebewesen ist sie anders, für die taube Schlange anders als für die Ultraschallspezialistin Fledermaus. Dazu ist diese Welt von Empfindung und Wahrnehmung nicht zu trennen. Überspitzt formuliert: Nicht Information, sondern Interpretation wird dem Gehirn geboten. Hier müssen die ersten zwei Probleme angesprochen werden:

1) Nur ein Teil der dem Gehirn angebotenen Information gelangt in unser Bewusstsein. Wie gross das Verhältnis der gesamten Information zu derjenigen ist, die ins Bewusstsein gelangt, wissen wir nicht, da wir den Zugriff auf das Unterbewusste nicht haben. Anhand des menschlichen Auges kann man aber grob (ohne Berücksichtigung von Zelldichte und Codierung) schätzen: Die gesamte beleuchtete Netzhautfläche eines Auges beträgt rund $1200 \mathrm{~mm}^{2}$. Der Ort der Aufmerksamkeitszuwendung, also die Stelle, mit welcher wir bewusst schauen («den Blick auf etwas richten»), anatomisch die Macula lutea, bestreicht eine Fläche von ca. $7 \mathrm{~mm}^{2}$. Grob geschätzt beträgt demnach die Fläche des bewussten Sehens 0,6\% der Gesamtfläche der gesehenen Umwelt. Für die Nase gilt: Bei jedem Atemzug, also etwa 12-15-mal pro Minute, gelangen Gerüche in die Nase. Wie oft wir aber etwas riechen, ist extrem variabel, zwischen zwei Wahrnehmungen können Stunden verstreichen. Ob diese Rechnung wirklich so stimmt, ist nicht sicher. Sicher ist, dass die Information, über die wir bewusst verfügen können, nur ein Bruchteil der gesamten Information beträgt. Und sicher ist, dass die im Unterbewussten abgelegte Information das Gehirn ebenfalls «beschäftigt» und damit die HI beeinflusst.

2) Das Problem des Geruchs. Von den 6 natürlichen Sinnen, die uns mit der Umwelt verbinden, ist der Geruchssinn der phylogenetisch älteste. Er wirkt möglicherweise am stärksten auf das Unterbewusste. Interessanterweise ist er ein Sinn, von dem es kein physikalisches Korrelat gibt. Wir können ihn nicht messen, weder qualitativ noch quantitativ. Auch die modernsten Olfaktometer (Geräte zum «Messen» des Geruchs) benutzen als Detektor die menschliche Nase. (Man verwechsle diesen Umstand nicht mit der Messung gewisser geruchsbildender Substanzen auf chemischem Wege. Hier wird die Konzentration einer chemischen Substanz gemessen, nicht ihre Eigenschaft des Geruchs.)

Die HI speist sich also aus Information (Geruch), die nicht zur Verfügung der Apparatur steht, die die KI erzeugt.

\section{Verarbeitung}

Was macht das Gehirn mit all dieser Information? Zunächst ermöglicht es dem Menschen, in und mit seiner Umwelt zurechtzukommen, indem es die Muskeln und Knochen in geordneter Weise in Aktion setzt. Wie das genau geschieht, entzieht sich zurzeit noch unserer Kenntnis. Wie oben erwähnt lässt sich die Verarbeitung jedoch am Ergebnis ablesen.

\section{Output - Aktuator}

Über das Zurechtkommen hinaus formt der Mensch seine Welt um und erweitert sie. Vorzugsweise sind hier Hände und Mundorgane im Spiel. Die Mundorgane ermöglichen die Entstehung von Sprache und Denken. Ein gutes Beispiel für die Hand ist der Knoten, die Verbindung mehrerer Schnüre zu einem Ganzen. So soll sich die Verbindung unter Zug nicht lösen, gleichzeitig soll sie rasch und einfach aufgelöst werden können (Schnürsenkel). Zwar ersinnt das Gehirn den Knoten, aber es ist die Hand, die es auf die Idee bringt. Die Interaktion von Gehirn und Hand geschieht wechselseitig, das Gehirn begreift, wie die Hand greift. Da- 
mit wird der Mensch zum Schöpfer. Es entstehen am Ende einer langen Entwicklung die grossen menschlichen Errungenschaften (Schöpfungen) Handwerk, Kunst und Wissenschaften.

Hier kommt, als drittes Problem, die Reihenfolge bei der Entstehung eines Werkes ins Spiel. Zuerst entsteht die Idee oder Imagination. Als Nächstes folgt die Ausführung, zuletzt die Überprüfung des Werkes. Am Ende geschieht die Prüfung oft durch Interpretieren und/oder Rechnen. In den Wissenschaften denke man etwa an die klassische Mechanik (Sir Isaac Newton und Emilie du Châtelet), die Kernspaltung (Otto Hahn und Lise Meitner) oder die Strukturanalyse der DNA (Rosalind Franklin und James Watson, Francis Crick). Wird diese Reihenfolge umgedreht, was technisch durchaus möglich ist - man kann schon lange Computer programmieren, die Musik im Stile von Palestrina komponieren -, so haftet dem Werk der Geruch des Beliebigen, Unechten an.

\section{Output - Geist}

Während Sensorik und Motorik im Verlauf der Zeit ihre optimale Funktion erreicht haben, schreitet die Entwicklung im Nervensystem fort. Das Gehirn entwickelt sich so weit, dass es mehr oder weniger unabhängig von Input und Output operieren kann. Man kann dies an Kindern beobachten, die mit einem Defekt der Sinnesorgane auf die Welt kommen. Sie erobern sich trotzdem unsere Welt. Eindrückliches Beispiel ist die taubblinde Helen Keller. Sie wurde eine Intellektuelle! Ohne Sensorik und Motorik entwickelt das Nervensystem kognitive Fähigkeiten, die hier unter dem Überbegriff «Geist» zusammengefasst werden. Einer dieser Begriffe ist das Unbekannte. Das Unbekannte schliesst nicht nur die Erkenntnis von Lücken ein - weissen Flecken auf der Karte des Wissens, das bekannte Unbekannte -, sondern auch das Nichtvorhandene, das unbekannte Unbekannte. Das Beispiel des Pulfrich-Effekts erläutert diesen Umstand: Lässt man ein Pendel mit einer weiss gestrichenen Kugel vor dunklem Hintergrund sauber horizontal hin- und herschwingen, dann wird man eindeutig erkennen können, dass die weisse Kugel sich nur in einer Ebene bewegt. Hält man nun vor ein Auge ein Graufilter (welches die Kugel etwas abdunkelt), so beobachtet man nach wenigen Sekunden eine elliptische Scheinbewegung der Kugel. Das Gehirn, welches ja weiss, dass es nur eine Kugel gibt, hat aus den zwei unterschiedlich hellen Signalen eine 3DBewegung "konstruiert». (Die Netzhautzapfen haben bei helleren Strukturen eine kürzere Latenzzeit bei der
Umwandlung der Energie in den Nervenimpuls als bei dunklen Strukturen.) Dieser Versuch ist nur nachvollziehbar, wenn das Pendel beidäugig betrachtet wird. Das eigentlich Bemerkenswerte daran ist seine Erfindung. Carl Pulfrich, ein deutscher Physiker, war seit Kindheit auf einem Auge blind. Er hat sich den Versuch theoretisch ausgedacht, ohne ihn aus der Umwelt oder Erfahrung ableiten zu können. Er konnte ihn auch nie selber beobachten!

Die Betrachtung der humanen Intelligenz aus der Sichtweise der Sinnesphysiologie zeigt einige Besonderheiten, die sie vom machine learning unterscheidet.

Erstens wurde sie in ihrer Entstehung durch die Sinnesorgane geprägt. Darüber hinaus speist sie sich aus Informationskanälen (Sinnen), die es beim machine learning nicht gibt.

Zweitens erfolgt die Informationsablage und -verarbeitung in zwei verschiedenen sich gegenseitig beeinflussenden Speichern.

Drittens ist bei der Entstehung ihres Produktes, des Werkes, die Reihenfolge bedeutsam. Zuerst Idee, dann Realisation, dann Überprüfung. Beim machine learning geht es genau umgekehrt. Unabhängig davon, wie die Daten zustande gekommen sind, wird zuerst gerechnet, werden Muster gelernt, dann erfolgt die Verifikation des Gelernten. (Ob daraus je eine Idee entsteht?)

Viertens erlaubt das Sichloslösen von den Sinnesorganen der humanen Intelligenz die Erschaffung neuer kognitiver Fähigkeiten, hier Geist genannt. Geist ist eine Form der Informationsverarbeitung im menschlichen Gehirn, die durch alleinige Rechenleistung nicht imitiert oder ersetzt werden kann. Dies würde höchstens dann denkbar werden, wenn bekannt ist, wie das Gehirn Bewusstsein und Unterbewusstsein schafft und steuert. Dadurch kommt der Mensch in die Lage, die Natur «verlassen» zu können, ohne wirklich aus ihr herauszutreten.

Diese und möglicherweise andere Besonderheiten verunmöglichen einen direkten Vergleich von machine learning zu humaner Intelligenz. Was aber nicht verglichen werden kann, kann sich auch nicht konkurrenzieren. Es wird spannend werden, die Entwicklung der KI in Zukunft zu verfolgen, und noch spannender wird es sein zu sehen, was die HI mit ihr alles anstellen wird.

\section{Verdankung}

Der Autor dankt Herrn Dr. phil. nat. Ph. C. D. Robert für die kritische Durchsicht des Manuskripts.

\section{Literatur}

1 Goldhahn J, Rampton V, Spinas GA. Could artificial intelligence make doctors obsolete? Schweiz Ärzteztg. 2019;100(8):242-4. 